\title{
Aircraft rerouting and rescheduling in multi- airport terminal area under disturbed conditions
}

\author{
Yuanyuan $\mathrm{Ma}^{1, *}$, Yuxin $\mathrm{Hu}^{1}$, He $\mathrm{Li}^{1}$, Yongjie $\mathrm{Yan}^{1}$, and Jianan $\mathrm{Yin}^{2}$ \\ ${ }^{1}$ State Key Laboratory of Air Traffic Management System and Technology, The 28th Research \\ Institute of China Electronics Technology Group Corporation, Nanjing 210007, P. R. China \\ ${ }^{2}$ National Key Laboratory of Air Traffic Flow Management, Nanjing University of Aeronautics and \\ Astronautics, Nanjing 211106, P. R. China
}

Keywords: Multi-airport system, Terminal area, Disturbed conditions, Aircraft rerouting, Aircraft scheduling, Multi-objective model, Evolutionary algorithm.

\begin{abstract}
The disturbances, caused by resource competition, weather changes, and demand uncertainties, frequently occur and result in obvious fluctuations (e.g., the increase or decrease of demand and capacity) in demand and capacity. This work addresses the problem of aircraft rerouting and rescheduling in presence of disturbances, characterized by the increase or decrease of demand and capacity, at a multi-airport terminal maneuvering area (TMA). A multi-objective optimization model is proposed to minimize the total flight delays and total extra flying time, considering the aircraft wake turbulence separation, runway operation rules, air traffic control regulations, scheduling time windows, and airport/airspace capacity, etc. Based on the non-dominated sorting genetic algorithm (NSGA-II), we designed an efficient evolutionary algorithm to solve the proposed model and search the Pareto solutions. Five scenarios are designed to analyze and compare the operation performance under normal and disturbed conditions, using the classic First-Come-First-Served (FCFS) and optimized scheduling (OPTS) methods. A case study is conducted for Shanghai Pudong and Hongqiao Airports, and the computational results show that, compared with the FCFS method, the proposed OPTS method significantly performs a overall better performance in minimizing total flight delay and total extra flying time, while slightly inferior in optimizing the delay and extra flying time of one single flight.
\end{abstract}

\section{Introduction}

The interactions among airports or flights have significant impacts on the TMA efficiencies. Spatial-temporal resource conflicts in a multi-airport terminal area frequently occur due to high volume demand, complex airspace structure, and dynamic operating environment. The movements of air traffic in TMA, and it can be described simply as: (i) arrivals, which fly into the TMA through a certain fix and then land on the runway system,

\footnotetext{
*Corresponding author: mayuanyuan2121@126.com
} 
and (ii) departures, which take off from the runway system and then fly out of the TMA through a certain fix, while considering appropriate priority settings. However, in some disturbed conditions such as bad weather, equipment failure, and general aviation influence, there probably are not enough capacity to accommodate as many arrivals and departures as normal. That leads a replace of the scheduled route of some flights to ensure a high efficiency for the whole-level operations. Hence, in order to conduct an integrated planning of TMA fixes and runways, and flexibly adjust the pre-set routes in complex airport environment, it is important to take full account of the abrupt decrease of airport capacity or increase of traffic demand at arrival / departure fixes.

A line of research focuses on the problem of aircraft routing and scheduling, and these two studies are taken simultaneously in an integrated model. The involved aircraft includes transportation flights [1] and unmanned aerial vehicles [2], and the modeling perspective covers airport surface and TMA with methods of directed graphs [3], minimum cost maximum flow [4], multi-commodity flow problem [5], Markov model[6], hybrid systems [7], Petri nets [8], etc. According the features of formulated models, various algorithms are designed to get the optimal or close-to-optimal solutions, such as $\mathrm{A}^{*}$ algorithm, genetic algorithm [9], Lagrange decomposition, and machine learning algorithm which is popular in recent years. Overall, most of the existing studies focus on the dynamic routing and scheduling and performance analysis in a single airport [10], considering adverse weather [11], and other important activities. However, the impacts caused by lack of shared resources, such as runway system, arrival fixes and departure fixes, and also the interactions among multiple airports in such cases are rarely considered simultaneously. In this work, we propose an efficient approach to address the problem of aircraft rerouting and rescheduling in multiairport TMA under disturbed conditions. In the rerouting part, the Pareto optimal route is selected for each flight, and the details of flight sequence and time at the fix and runway system are calculated in the rescheduling part.

The outline of this paper is as follows. Section 2 presents a multi-objective optimization model of aircraft rerouting and rescheduling under disturbed conditions. Then an evolutionary algorithm is designed to get the Pareto solutions of the proposed integrated model. In Section 3, we conduct a real-world case study for Shanghai multi-airport TMA, and illustrate the computational results. Finally, the findings and insights are discussed in Section 4 by analyzing the computational results.

\section{Method}

\subsection{OPTS model}

Consider a set of airports A, which are covered in a multi-airport TMA system. Let $\mathrm{F}$ be the set of arrival and departure flights. For any airport $i \in \mathrm{A}, \mathrm{F}_{i}$ denotes the corresponding flights set. In this section, we propose the OPTS model which have two objectives as Eq. 1 minimizes the total flight delay time and Eq. 2 minimizes the extra flying time, where $\delta_{i}$ is the delay time of flight $i$ and $\delta_{i}=\left|s_{i}-t_{i}\right| . s_{i}$ and $t_{i}$ respectively represent the assigned and scheduled runway time. Particularly, negative delay is treated as the same as positive delay in this paper. $e_{i}$ is the extra flying time of flight $i$, which can be divided into two parts, the extra times outside $\left(\Lambda_{i}\right)$ and inside $\left(\Delta_{i}\right)$ the TMA, and $e_{i}=\Lambda_{i}+\Delta_{i}$. For arrival flights, the extra flying time outside the TMA $\Lambda_{i}$ is consisted of the extra flying time for changing STAR and the waiting time outside the TMA for the proper time to enter, which is equivalent to the difference between estimated time $\mathrm{r}_{i}$ and assigned time $\mathrm{u}_{i}$ of arrival flight $i$ flying through the fix. 


$$
\begin{aligned}
& \min \left(\sum_{i \in F} \delta_{i}\right) \\
& \min \left(\sum_{i \in F} e_{i}\right)
\end{aligned}
$$

s.t.

$$
\begin{gathered}
s_{j} \geq \varepsilon_{i j} \omega_{i j}\left(s_{i}+\kappa_{i j}\right), \varepsilon_{i j}, \omega_{i j} \in\{0,1\}, s_{i}, s_{j}, \kappa_{i j} \geq 0, \forall i, j \in F \\
s_{j} \geq \lambda_{i j} \varepsilon_{i j} \gamma_{i j}\left(s_{i}+\varphi_{i j}\right), \lambda_{i j}, \varepsilon_{i j}, \gamma_{i j} \in\{0,1\}, s_{i}, s_{j}, \varphi_{i j} \geq 0, \forall i, j \in F \\
s_{j} \geq \vartheta_{i j} \varepsilon_{i j} \gamma_{i j}\left(s_{i}+\phi_{i j}\right), \vartheta_{i j}, \varepsilon_{i j}, \gamma_{i j} \in\{0,1\}, s_{i}, s_{j}, \phi_{i j} \geq 0, \forall i, j \in F \\
s_{j} \geq \lambda_{i j} \varepsilon_{i j} s_{i j}\left(s_{i}+\psi_{i j}\right), \psi_{i j}=E / V_{j}, \lambda_{i j}, \varepsilon_{i j}, \varsigma_{i j} \in\{0,1\}, s i, s j, E, V_{j} \geq 0, \forall i, \\
j \in F \\
s_{j} \geq \lambda_{i j} \varepsilon_{i j}\left(s_{i}+\sigma_{i}\right), \lambda_{i j}, \varepsilon_{i j} \in\{0,1\}, s_{i}, s_{j}, \sigma_{i} \geq 0, \forall i, j \in F \\
u_{j} \geq \eta_{i j} \varepsilon_{i j} \rho_{i j}\left(u_{i}+\varrho_{i j}\right), \eta_{i j}, \varepsilon_{i j}, \rho_{i j} \in\{0,1\}, s_{i}, u_{j}, \varrho_{i j} \geq 0, \forall i, j \in F \\
\alpha_{i} \leq s_{i} \leq \beta_{i}, \alpha_{i}, \beta_{i} \geq 0, \forall i \in F \\
\left(p_{i}, q_{i}\right) \in Y_{i}, p_{i}, q_{i} \geq 0, \forall i \in A \\
u_{i}=\min \left(w_{i j}\right), w_{i j} \geq r_{i}+x_{i j}, u_{i}, w_{i j}, r_{i}, x_{i j} \geq 0, \forall i \in F, j \in Z
\end{gathered}
$$

Eq. 3 is the wake turbulence separation, which is set based on the type of flights, considers the impact of preceding flight's wake on the succeeding flights, where $\kappa_{i j}$ represents the minimum wake turbulence separation to be met between flights $i$ and $j$. $\varepsilon_{i j}$ is a decision variable which equals to 1 when the assigned runway time of arrival (departure) flight $i$ is earlier than that of arrival (departure) flight $j$, and zero otherwise. $\omega_{i j}$ is also a decision variable, which equals to 1 when both $i$ and $j$ are arrival (departure) flights, zero otherwise. Eq. 4 and Eq. 5 refers to two consecutive arrival flights using the same and adjacent instrument landing system (ILS), where $\lambda_{i j}$ and $\vartheta_{i j}$ are decision variables, which equal to 1 when flights $i$ and $j$ are assigned to use the same runway and two adjacent runways, and zero otherwise. $\gamma_{i j}$ is a decision variable, which equals to 1 when flights $i$ and $j$ are both arrival flights, and zero otherwise. $\varphi_{i j}$ is the time separation of the two arrival flights $i$ and $j$ using the same ILS. $\phi_{i j}$ is the time separation of the two consecutive landing flights using two adjacent ILSs. Eq. 6 is another constrain for the two flights using the same runway should satisfy when the preceding one is a departure flight and the succeeding one is an arrival flight. $\psi_{i j}$ is the time separation between flight $i$ and $j$ using the same runway. $\varsigma_{i j}$ is a decision variable, which equals to 1 when the preceding one is a departure flight and the succeeding one is an arrival flight, and zero otherwise. $E$ is the interception distance. $V_{j}$ is the speed of the succeeding flight. Eq. 7 ensures that there is only one flight using runway at the same time, where $\sigma_{i}$ is the runway occupancy time of the preceding flight $i$. Eq. 8 ensures two consecutive flights flying into the TMA through the same fix satisfy the ATC transfer separation, where $u_{i}$ is the time of flight $i$ flying through fix. $\varrho_{i j}$ is the time separation of the two consecutive flights $i$ and $j$ flying into the TMA through the same fix, $\eta_{i j}$ is a decision variable, which equals to 1 
when flight $i$ and $j$ fly through the same fix, and zero otherwise. $\rho_{i j}$ is a decision variable, which equals to 1 when flight $i$ fly through the fix ahead of flight $j$, and zero otherwise. Eq. 9 ensures the runway time of each flight is feasible, where $\alpha_{i}, \beta_{i}$ are the earliest and latest time of the flight $i$ arriving at fix or taking off. Eq. 10 ensures the quantity of served arrival and departure flights should satisfy the limit of the airport capacity envelope, where $Y_{i}$ is the set of all possible served flight quantity pairs of arrival quantity $p_{i}$ and departure quantity $q_{i}$ for airport $i$. Eq. 11 refers to the time of flying through the replaced fix is set to be earlier than that through the planned fix, which is aimed to make sure that it is useful to adjust the STAR/SID and maximize the utilization of restricted capacity of TMA, where $w_{i j}$ is the possible time that flight $i$ fly through the fix $j$.

\subsection{Algorithm}

Considering the described OPTS is a multi-objective optimization problem, in this section we design an improved evolutionary algorithm to get the close-to-optimal Pareto solutions of the rerouting and rescheduling problem, based on the non-dominated sorting genetic algorithm (NSGA-II). The Pareto solutions are significantly useful for the stakeholders to make tradeoffs between the total flight delay time and the extra flying time. The implementation procedure of our evolutionary algorithm is as follows:

Step 1: Time coding scheme is chosen in our algorithm design and the outputs of FCFS strategy is set as the initial parent population $\mathrm{P}_{0}$ to improve calculation efficiency. After fast non-dominated sorting strategy is performed on the population $\mathrm{P}_{0}$, the dominant parameters of each individual in the population is determined while the generation No. of population $g$ $=0$.

Step 2: Apply a series of operators including binary tournament selection, crossover, and mutation on the population $\mathrm{P}_{0}$, the offspring population $\mathrm{Q}_{0}$ is formulated with the size $\left|\mathrm{P}_{0}\right|$ $=\left|\mathrm{Q}_{0}\right|$.

Step 3: Combine the parent population and the offspring population in the current evolutionary generation $\mathrm{R}_{g}=\mathrm{P}_{g} \cup \mathrm{Q}_{g}$ then use the non-dominated sorting operator to generate a series of Pareto fronts at different levels $\left\{\mathrm{F}_{s} \mid \mathrm{s}=1,2,3, \ldots\right\}$.

Step 4: Crowding comparison operator is used to sort the crowded distances which is calculated for each layer of Pareto front $\mathrm{F}_{s}$, and the best $\left|\mathrm{Q}_{g}\right|$ individuals is selected to form the population $\mathrm{P}_{g+1}$.

Step 5: Binary tournament selection, crossover, and mutation operators are performed on the $g+1$ th operations, and the population $\mathrm{Q}_{\mathrm{g}+1}$ is formed of which the size $\left|\mathrm{P}_{\mathrm{g}+1}\right|=\left|\mathrm{Q}_{\mathrm{g}+1}\right|$.

Step 6: If the current evolution process satisfies the termination condition (e.g., maximum generation No.), the algorithm terminates and the Pareto optimal solutions will be output; otherwise, let $g=g+1$ and go to Step 3 for the next iteration.

\section{Results}

In this paper, we conduct a case study of aircraft rerouting and rescheduling under disturbed conditions of capacity and demand, for Shanghai multi-airport TMA, which is a typical metroplex terminal area in the world including Pudong Airport and Hongqiao airport. The TMA airspace structure of Shanghai multi-airport system is illustrated in Fig. 1. The fixes in terminal area are shared by both Pudong and Hongqiao airport, but the fix BK is only available for the arrival flights of Pudong airport for its higher demand than Hongqiao airport. And the fix SASAN can be used by both arrival and departure flights, and it is dealt as two fixes SASAN-A and SASAN-D in this paper. 


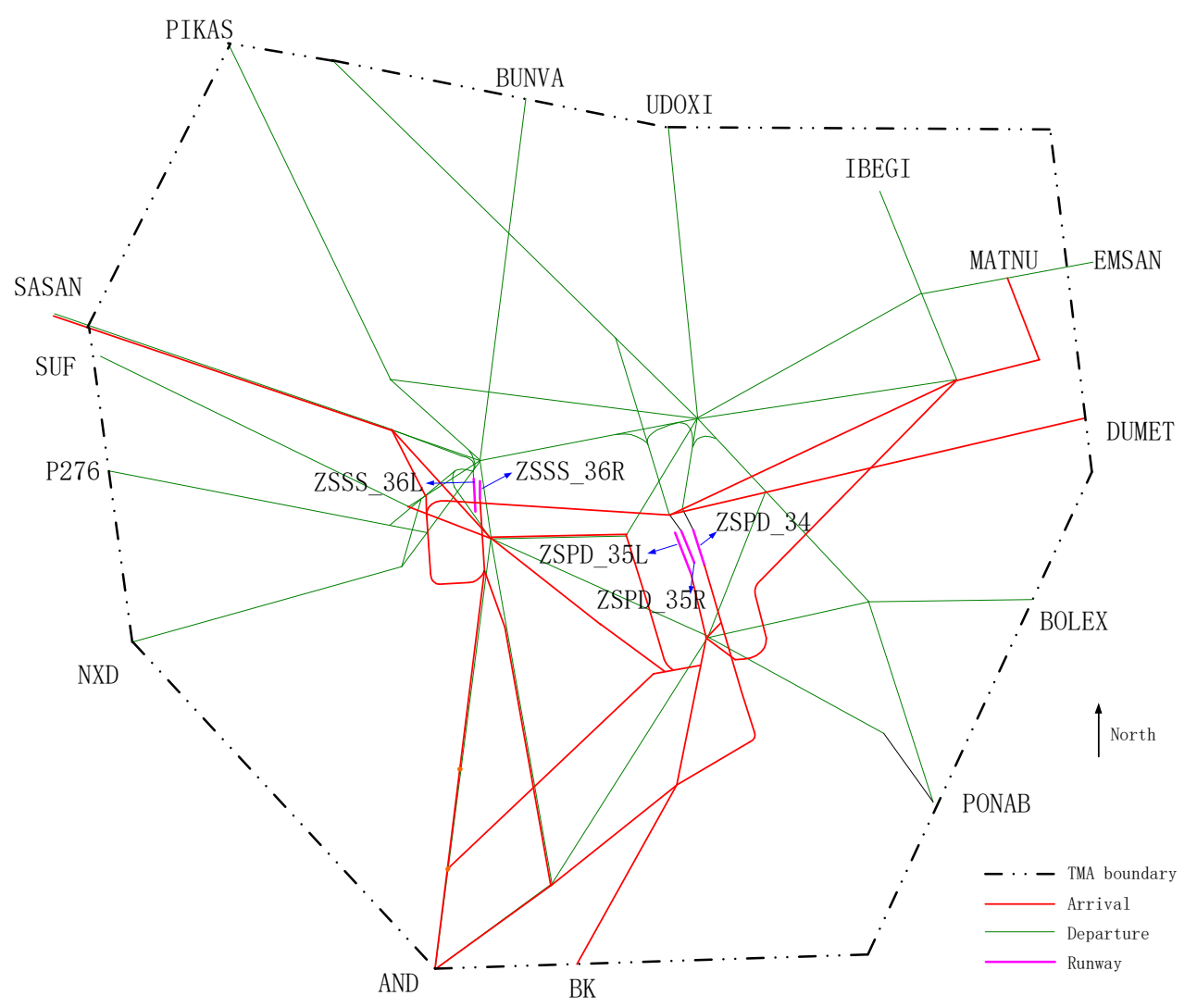

Fig. 1. Structure of Shanghai multi-airport TMA.

\subsection{Scenario design}

The total number of aircraft with the planning horizon 15:00-16:59 is 108. In order to analyze and compare the results under different disturbed conditions at the same time, we design 5 scenarios based on the changes of capacity and demand of two fixes SASAN and PIKAS.

Scenario A: Normal condition. The airspace capacity and traffic demand are not unaffected by obvious influencing factors within the planning horizon.

Scenario B: Decreased capacity of arrival fix. The arrival fix SASAN-A is selected to generate a scenario where the capacity will be decreased by $40 \%$.

Scenario C: Decreased capacity of departure fix. Similar with Scenario B, the departure fix PIKAS is selected to generate a scenario where the capacity will be decreased by $40 \%$.

Scenario D: Increased arrival flights. Assume that the arrival flights scheduled to fly through fix SASAN-A in the former interval (an hour) were delayed (i.e., added) to the time interval with the planning horizon.

Scenario E: Increased departure flights. Similar with Scenario D, the departure flights scheduled to fly through fix PIKAS in the former interval (one hour) were delayed (i.e., added) to the time interval with the planning horizon.

The population size in Scenarios A E is 200, the maximum evolutional generation No. is 600 in Scenarios A D while 800 in Scenarios E. The coding length is 108, the crossover probability is 0.8 , and the mutation probability is 0.015 . The rules of linear recombination and random factor mutation are used to perform genetic operators. 


\subsection{Computational results}

\subsubsection{Results in Scenario $A$}

The results in Scenario A show that the individuals of evolution population gradually approach the optimal direction and quickly get the Pareto optimal solutions in the decision space with the increase of iterations. Take the flights of Pudong and Hongqiao airports as an example, the running time of our algorithm is about 10 minutes including the data processing, solution seeking and result visualization. Note that multiple tests also show the good quality of our algorithm. Simulation results show that the initial population converged gradually after 400 iterations, the Pareto optimal solutions shown in Fig. 2 can be got with the diversity of population. The minimum flight delay and extra flying time using the proposed OPTS method are $6409 \mathrm{~s}$ and $6105 \mathrm{~s}$, respectively. In the following part, the results of Pareto solution (6501, 6229) from OPTS is chosen to compare with that from the FCFS method.

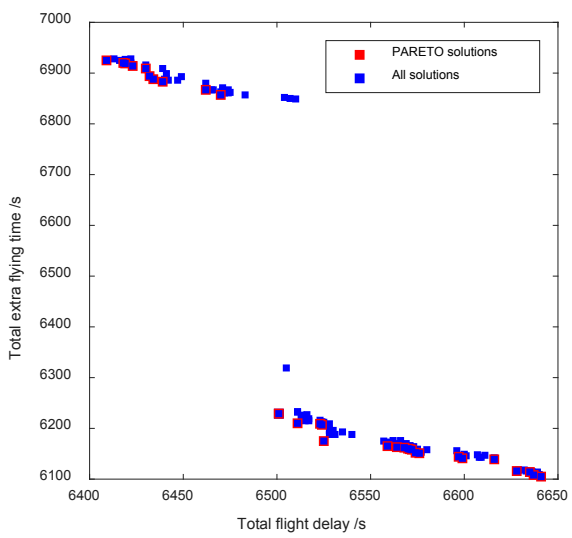

Fig. 2. Distribution of Pareto solutions.

Fig. 3 shows the comparison of flight delay time using two methods of FCFS and OPTS. The red and blue bars correspond to the results of FCFS and OPTS, respectively. The color of the top of each bar indicates the method which has the larger delays.

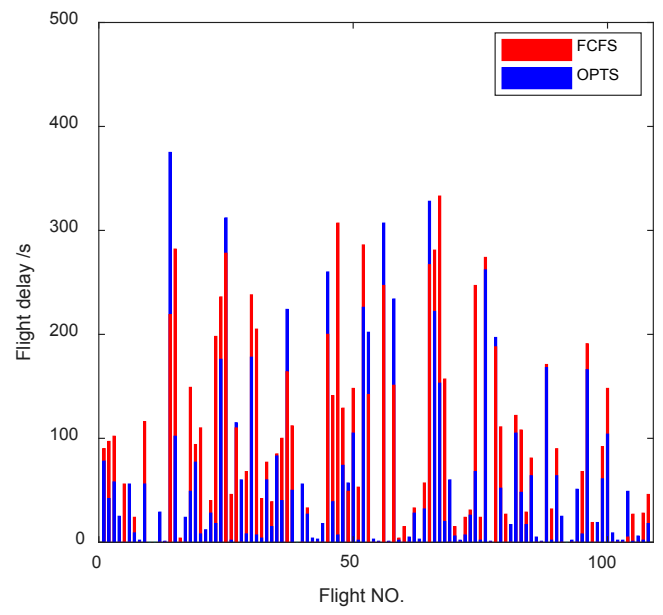

Fig. 3. Comparison of flight delays using FCFS and OPTS methods. 
It can be seen from Fig. 4 that most of the flights have less delay by using the OPTS method than that of FCFS, and the total flight delay under OPTS is $6501 \mathrm{~s}$, with a $24.5 \%$ decrease compared to that under FCFS. The mean and the maximum delay under these two methods are $60.2 \mathrm{~s}$ and $375 \mathrm{~s}, 79.7 \mathrm{~s}$ and $333 \mathrm{~s}$. Note that there is only one flight's delay time from OPTS with a value of more than 333s. There are 61, 43 and 4 flights under OPTS of which the delay time is less than, more than and equal to that under FCFS. And no flights have the same delay under two methods.

Fig. 4 shows the comparison of extra flying time using the FCFS and OPTS methods. It can be seen that most of the flights has less extra flying time under OPTS than that under FCFS, but part of the studied flights under OPTS has larger extra flying time than that under FCFS. The total extra flying time under OPTS is $6229 \mathrm{~s}$, with a $30.1 \%$ decrease compared to that under FCFS. The mean and maximum extra flying time under these two methods are $57.7 \mathrm{~s}$ and $375 \mathrm{~s}, 82.5 \mathrm{~s}$ and $333 \mathrm{~s}$. Note that there is only one flight of which the extra flying time calculated by OPTS is more than 333s. In addition, there are 53, 20 and 35 flights' extra flying time are less than, more than and equal to that under FCFS.

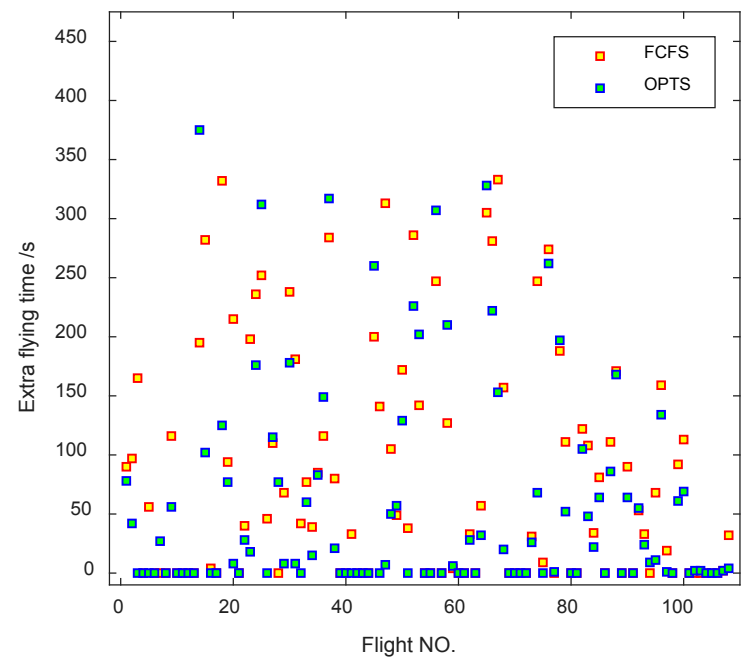

Fig. 4. Comparison of flight extra flying time using OPTS and FCFS methods.

From Fig. 3 and Fig. 4, we can see that OPTS method had better optimization performance on the overall flights, but a bit worse on one single flight than the FCFS method. The reason is that the OPTS method proposed in this paper focuses on the overall improvement of flight performance at the expense of sacrificing the performance of a certain flight. It can be also concluded that OPTS have a better optimization effect in minimizing extra flying time than minimizing flight delay, which probably because there exists some inherent delay due to the unreasonable flight plan made in the strategic level.

In addition, the extra flying time is significantly related to STAR/SID and obviously changes as STAR/SID is adjusted, which is probably another reason that OPTS has more effects in minimizing the extra flying time than minimizing the flight delay besides the reason presented above. Also, OPTS has a better effect on the flights at Pudong airport which holds 3 runways (one for arrival, one for departure, and the third one is for both arrival and departure) than that at Hongqiao airport which holds 2 runways (one for arrival and the other is for departure). We can find that the airport with more runways is more competitive in occupying the TMA airspace resources, because generally more runways can also accommodate more air traffic movements. 
The allocation of arrival and departure fixes are illustrated in Fig. 5 and Fig. 6. We can see that few flights change the STAR/SID under Scenario A in which no special events affect the TMA operations. The distance between departure fixes IBEGI and UDOXI is close to each other, which makes the change of fix costs a small extra flying time. That is to say, the estimated time over the new point is earlier than that over the original point. Additionally, the SID/STAR structure shows that the distribution of departure routes are more flexible and decentralized than that of arrival routes, and then the change of departure fix may improve the flight efficiency in TMA.

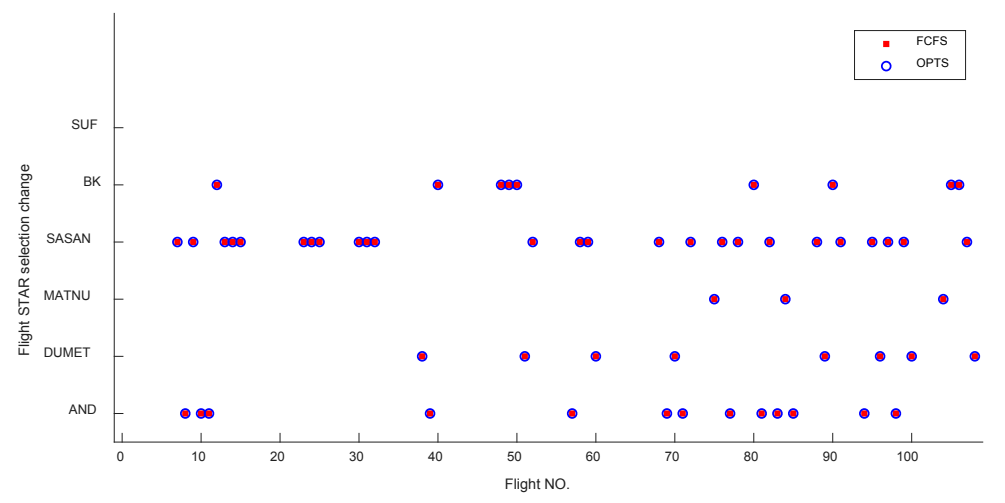

Fig. 5. Allocation of arrival fixes.

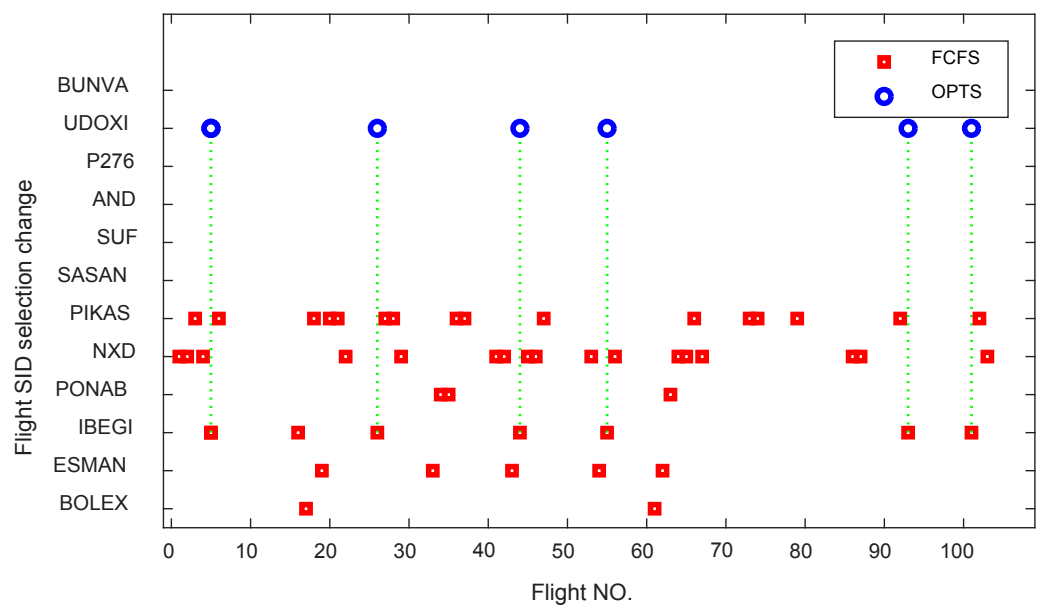

Fig. 6. Allocation of departure fixes.

From Fig. $2 \sim$ Fig. 6 , we can obviously see that the OPTS is more effective than the FCFS especially in optimizing the overall performance of all the flights in Shanghai multi-airport TMA system. Airport with multiple runways has some advantages in the utilization of key airspace resources. In addition, it is found that the OPTS has a slightly and not obviously better effect in optimizing the departure flights than arrival flights, with the reason that there are more divergent SIDs than STARs which lead to more choices and better effect in aircraft and rescheduling for departure flights than that for arrival flights. 
For the simplicity reason, the results in Scenarios $\mathrm{B} \sim \mathrm{E}$, which is similar with the result in Scenario A, are no longer described in detail. Hence, we conduct a comprehensive analysis and comparison of flight delay time and extra flying time results in Scenarios A E. The comparison results are illustrated in Table 1 2. It can be found that the effect of OPTS on arrivals is less than that on departures, which is probably because the resource available for each arrival flights decreases when the arrival capacity is decreased or arrival flights are increased as mentioned in Scenario B and Scenario D, compared with the results in Scenario A. We can also see that the airport with more resources (e.g., runways, taxiway segments and gates) has more advantages in dealing with dynamic changes of capacity and demand. The departures of Pudong Airport with more runways and more resources are better optimized than the departures of Hongqiao Airport using OPTS in Scenario B and Scenario D under disturbances of less arrival capacity or more arrival traffic. In addition, the effect on the departures in Scenario D is little less than that in Scenario B, because more arrivals means more shared resource utilization prior to departures under certain conditions of arrival capacity.

Table 1. Comparison of total flight delay time using OPTS and FCFS in different Scenarios.

\begin{tabular}{|c|c|c|c|c|c|c|c|c|c|c|c|}
\hline \multirow{3}{*}{ Scenarios } & \multicolumn{2}{|c|}{$\begin{array}{l}\text { Total flight delay } \\
\text { /s }\end{array}$} & \multicolumn{9}{|c|}{$\begin{array}{l}\text { Total flight delay reduction under OPTS } \\
\text { compared to that under FCFS (\%) }\end{array}$} \\
\hline & \multirow{2}{*}{ FCFS } & \multirow{2}{*}{ OPTS } & \multirow{2}{*}{ Total } & \multirow{2}{*}{ Arr } & \multirow{2}{*}{ Dep } & \multirow{2}{*}{$\mathrm{PD}$} & \multirow{2}{*}{ HQ } & \multicolumn{2}{|c|}{ PD } & \multicolumn{2}{|c|}{ HQ } \\
\hline & & & & & & & & Arr & Dep & Arr & Dep \\
\hline Scenario $A$ & 8612 & 6501 & 24.5 & 23.6 & 25.6 & 27.8 & 21.2 & 25.8 & 34.5 & 18.7 & 22.5 \\
\hline Scenario B & 23891 & 12622 & 46.2 & 49.77 & 30.6 & 33.9 & 58.8 & 31.4 & 52.4 & 71.4 & 19.6 \\
\hline Scenario $C$ & 8612 & 6473 & 24.8 & 26.3 & 23.0 & 25.8 & 23.9 & 25.5 & 27.1 & 28.2 & 21.7 \\
\hline Scenario D & 113320 & 100533 & 11.3 & 11.0 & 17.6 & 5.6 & 18.7 & 4.7 & 42.3 & 19.6 & 4.7 \\
\hline Scenario E & 72094 & 69235 & 5.4 & 31.3 & 3.5 & 5.9 & 4.9 & 32.2 & 2.7 & 29.3 & 4.1 \\
\hline
\end{tabular}

Table 2. Comparison of total extra flying time using OPTS and FCFS in different Scenarios.

\begin{tabular}{|c|c|c|c|c|c|c|c|c|c|c|c|}
\hline \multirow{3}{*}{ Scenarios } & \multicolumn{2}{|c|}{$\begin{array}{c}\text { Total extra flying } \\
\text { time } / \mathrm{s}\end{array}$} & \multicolumn{9}{|c|}{$\begin{array}{c}\text { Total extra flying time reduction under OPTS } \\
\text { compared to that under FCFS }(\%)\end{array}$} \\
\hline & \multirow{2}{*}{ FCFS } & \multirow{2}{*}{ GA } & \multirow{2}{*}{ Total } & \multirow{2}{*}{ Arr } & \multirow{2}{*}{ Dep } & \multirow{2}{*}{ PD } & \multirow{2}{*}{ HQ } & \multicolumn{2}{|c|}{ PD } & \multicolumn{2}{|c|}{ HQ } \\
\hline & & & & & & & & Arr & Dep & Arr & Dep \\
\hline Scenario $A$ & 8909 & 6229 & 30.1 & 26.1 & 33.8 & 34.9 & 25.7 & 24.9 & 54.7 & 28.4 & 24.4 \\
\hline Scenario B & 24422 & 14337 & 41.3 & 40.6 & 44.0 & 23.4 & 59.2 & 13.4 & 75.0 & 71.6 & 24.7 \\
\hline Scenario $C$ & 12069 & 7596 & 37.1 & 30.1 & 40.9 & 31.6 & 41.9 & 26.7 & 36.6 & 36.8 & 43.5 \\
\hline Scenario D & 45123 & 33825 & 25.0 & 24.1 & 32.1 & 10.1 & 42.7 & 4.8 & 66.8 & 48.7 & 8.7 \\
\hline Scenario E & 75682 & 70030 & 7.5 & 36.6 & 5.7 & 6.7 & 3.4 & 4.0 & 8.0 & 41.4 & 7.0 \\
\hline
\end{tabular}

According to the comparison results between Scenario B and Scenario A, the optimization effect on departure scheduling is better when arrival capacity is decreased in Scenario B. Otherwise, the effect is influenced when arrival flights is increased by comparing the results of Scenario D and Scenario A. Note that it is unfair but reasonable that there is little or no influence of the optimization effect on arrival flights scheduling when departure capacity is decreased according to the results comparison of Scenarios D, E and A. 
The results in Scenario A shows that OPTS effect on flights rerouting and rescheduling of Pudong airport is better than that of Hongqiao airport. However, we can also find that the optimization effect on the departure flights of Pudong is better than that of Hongqiao airport when arrival capacity is decreased, and the influence on departure flights of Pudong airport is less than that of Hongqiao airport when the arrival flights is increased.

A significant difference is that the effect of OPTS on arrivals is not influenced when departure capacity is decreased in Scenario $\mathrm{C}$ and departure demand is increased in Scenario E. It is almost same with the effect on departures when arrival capacity is decreased in Scenarios B and arrival demand is increased in Scenario D. That's probably due to the priority of arrivals compared to departures in resource allocations. It can be also indicated that more flights of Pudong airport than the flights of Hongqiao airport are rerouted and rescheduled by OPTS which may because more runways and STARs cause more flexibility in the selection of aircraft rerouting and rescheduling. Also, there are more flights of Pudong than that of Hongqiao airport, according to flight plan.

\section{Conclusions}

This paper studies the problem of aircraft rerouting and rescheduling in presence of increase or decrease of demand and capacity. We propose a multi-objective optimization model OPTS and design an improved evolutionary algorithm based on the NSGA-II algorithm. Five scenarios are designed to reflect the disturbed conditions. And the case study for Shanghai multi-airport TMA operations shows a series of interesting conclusions which is illustrated as follows.

The proposed OPTS method has a better overall performance in minimizing total flight delay and extra flying time than FCFS, but slightly worse in optimizing the delay and extra flying for some single flights. The OPTS is characterized by scarifying the performance of some flights to get an improvement for overall flights. Comparing the optimization effect of departures with that of arrivals, there is slightly and not obviously better effect using the proposed OPTS method. From the comparisons of all the five designed scenarios, it reveals a fact that an airport with more runways has a more competitive ability in the utilization of shared resources in TMA than the airports with less runways. Changing the selection of STAR or SID of flights can partly alleviate the flight delay and airspace congestion caused by the imbalance between capacity and demand of resources.

The significance of our work is a new optimization model and evolutionary algorithm of aircraft rerouting and scheduling in multi-airport TMA, which brings significant benefits to the high density traffic operations at metroplex systems. The findings can provide some references about multi-airport operations on the aspects of enhancing the performance metrics of delay and flying time.

This work was supported by the Natural Science Foundation of Jiangsu Province (Grant Nos. BK20190416, BK20170157), China Postdoctoral Science Foundation (Grant No. 2017M611809), Jiangsu Planned Projects for Postdoctoral Research Funds (Grant No. 1701099C) and National Key Technologies R\&D Program of china (Grant No. 2017YFB0503401).

\section{References}

1. F. Wieland, A. Tyagi, V. Kumar, W. Krueger, METROSIM: a metroplex-wide route planning and airport scheduling tool, 14th AIAA Aviation Technology, Integration, and Operations Conference. 2014. 
2. R. LIU, F. YANG, H. ZHANG, Path planning for UAV based on improved chaotic and colony algorithm(CACA), Command information system and technology. 2018, 9(6): 41-48.

3. X. Zhu, Research on Petri Nets-based aircraft routing and conflict control for A-SMGCS, Nanjing University of Aeronautics and Astronautics. 2012.

4. J. G. Herrero, A. Berlanga, J. M. Molina, J. R. Casar, Methods for operations planning in airport decision support systems, Applied Intelligence. 2005, 22(3): 183-206.

5. K. Song, The Research of rerouting problem in air traffic flow management, Nanjing University of Aeronautics and Astronautics. 2002.

6. V. Singh, K. E. Willcox, Methodology for path planning with dynamic data-driven flight capability estimation, 17th AIAA/ISSMO Multidisciplinary Analysis and Optimization Conference. June 2016, Washington D.C., 13-17

7. X. Tang, H. An, C. Wang, Conflict avoidance oriented airport surface aircraft taxiing guidance method, Journal of Southwest Jiaotong University. 2011, 46(6): 1032-1039.

8. X. Zhu, X. Tang, S. Han, Aircraft initial taxing route planning based on petri net and genetic algorithm, Journal of Southwest Jiaotong University. 2013, 48(3): 565-573.

9. Y. Ma, Hu M, J Yin, Tian W, Collaborative sequencing and scheduling method for arrival and departure traffic flow in multi-airport terminal area, Acta Aeronautica et Astronautica Sinica. 2017, 38, 320222-1-320222-13.

10. J. Yin, M. Hu, Y. Ma, K. Han, D. Chen, Airport taxi situation awareness with a macroscopic distribution network analysis, Networks and Spatial Economics. 2019, 19, 669-695.

11. M. ZHANG, Y. SUN, Y. WANG, Temporal and spatial characteristic analysis for slot hop during flight departure, Command information system and technology. 2019, 10(1): 43-65. 